\title{
Influence of the picosecond defect distribution on damage accumulation in irradiated $\alpha$-Fe
}

\author{
C. Björkas, ${ }^{1,2}$ K. Nordlund, ${ }^{1}$ and M. J. Caturla ${ }^{3}$ \\ ${ }^{1}$ EURATOM-Tekes, Department of Physics, P.O. Box 43, FI-00014 University of Helsinki, Finland \\ ${ }^{2}$ Institute of Energy and Climate Research - Plasma Physics, Forschungszentrum Juelich GmbH, \\ Association EURATOM-FZJ, Partner in the Trilateral Euregio Cluster, Juelich, Germany \\ ${ }^{3}$ Departamento de Fisica Aplicada, Universidad de Alicante, E-03690 San Vicente del Raspeig, Spain \\ (Received 28 June 2011; revised manuscript received 7 December 2011; published 9 January 2012)
}

\begin{abstract}
The importance of the defect distribution produced in the first few picoseconds of a collision cascade on long-term damage evolution is studied with molecular dynamics and kinetic Monte Carlo (KMC) methods. Three different interatomic potentials are used to obtain the primary damage produced by energetic recoils in $\alpha$-Fe. Contrary to previous results, a dependence of cluster-size distribution with recoil energy is obtained. Moreover, large variations in this distribution are observed depending on the interatomic potential. Using the results for $50 \mathrm{keV}$ collision cascades, damage accumulation is modeled with KMC. The accumulation rate of damage visible under transmission electron microscopy predicted by KMC depends significantly on the database used for cascade damage and, therefore, on the interatomic potential. Based on these results, we show that the comparison of cluster-size distributions with experiments can be used to test the reliability of interatomic potentials.
\end{abstract}

DOI: 10.1103/PhysRevB.85.024105

PACS number(s): 28.52.Av, 28.52.Fa, 61.72.J-

\section{INTRODUCTION}

Irradiation of a material produces lattice defects, which can lead to significant alterations of its properties. This is critical for materials designed for an environment where bombardment of high-energy particles is frequent and unavoidable, such as the steel constructions of a fusion reactor. The understanding of the production of the lattice defects and their relation to, for instance, irradiation-induced swelling and embrittlement is of vital importance for the construction of a safe and long-lived reactor.

Defect production is clearly a multiscale phenomenon, going from the formation of individual collision cascades on the picosecond scale up to years of evolution of the defects. No single method capable of modeling the whole range of time and length scales is available, thus, the combination of simulation techniques offers a solution at hand. For instance, molecular dynamics (MD) simulations can be used to model the primary damage produced in collision cascades, and kinetic Monte Carlo (KMC) methods can model the further evolution of that primary damage. In the scope of such "multiscaling," one important issue has been addressed by several authors ${ }^{1,2}$ with no definite answer yet: how sensitive is the long-term evolution of the damage to the specific features of the defect distribution obtained on picosecond time scales?

In the first molecular dynamics calculations of collision cascades in the heat-spike regime, the importance of the primary damage distribution has already been pointed out. ${ }^{3}$ Models of defect evolution were then modified to take into account the clustering of defects obtained in MD calculations. This is the case of the "production bias" model ${ }^{4}$ that included defect clustering in order to explain swelling rates measured experimentally in pure metals at low dose..$^{5}$ More recently, a combination of MD and KMC has shown that differences in the concentration of defects measured experimentally using transmission-electron microscopy (TEM) in irradiated Fe and $\mathrm{Cu}$ can be attributed to differences in the cascade defect distribution: MD calculations show that defects in $\mathrm{Cu}$, both self-interstitials and vacancies, are typically in the form of clusters, whereas in $\mathrm{Fe}$, vacancies are mostly isolated and self-interstitials are in clusters. ${ }^{6,7}$ Since only clusters above a certain size, i.e., $1-2 \mathrm{~nm},{ }^{8,9}$ are visible under TEM, vacancy clusters can be clearly observed in $\mathrm{Cu}$, while this is not the case in $\mathrm{Fe}$.

Previous studies consistently show that a random distribution of Frenkel pairs and a distribution of defects, as obtained from MD simulations, will lead to very different damage evolution. ${ }^{2,10,11}$ However, how much the long-term evolution of defects is affected by the specific features of the cascade damage is still under debate. This is an important issue since this distribution is obtained from molecular dynamics simulations using empirical potentials. Many different iron potentials have been, and are being, developed and tested but, to date, no "perfect" or "most reliable" potential has emerged. The difficulty of that selection lies mainly in the absence of reference cases or experimental observations to compare to. Hence, a cascade feature that can directly be compared to experiments would improve the situation.

Recent simulations by Becquart and co-workers ${ }^{2,12,13}$ have shown that the resulting damage in Fe depends mostly on the KMC parameters used in the simulation and not on the initial damage distribution obtained from different interatomic potentials. From these studies, the authors conclude that a binary collision approximation (BCA), with the appropriate recombination radius for vacancies and self-interstitials, can be used to model primary damage in pure $\mathrm{Fe}$, since, according to their calculations, cluster-size distribution is independent of cascade energy. ${ }^{14}$ Therefore, they were able to exclude cascade properties that depend on the primary knock-on atom (PKA) energy, such as subcascade morphology and point-defect spatial correlations, from the list of possible key features affecting the outcome. The only property identified as a PKA energy invariant was the initial cluster-size distribution, which therefore might influence the cluster growth.

Primary damage in the above studies was produced by lowenergy cascades, making the formation of large defect clusters improbable and no clusters above $\sim 20$ defects were observed 


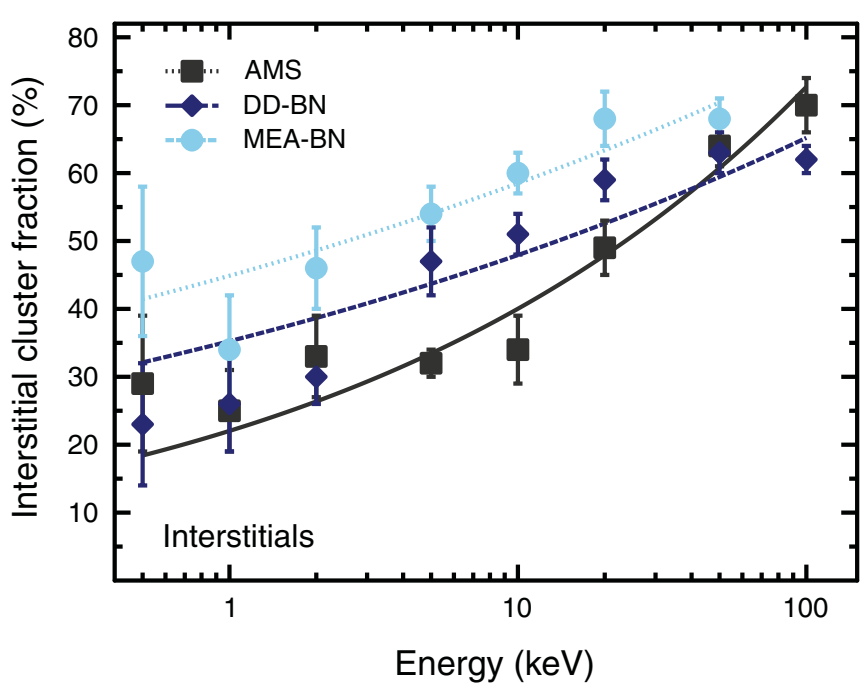

(a)

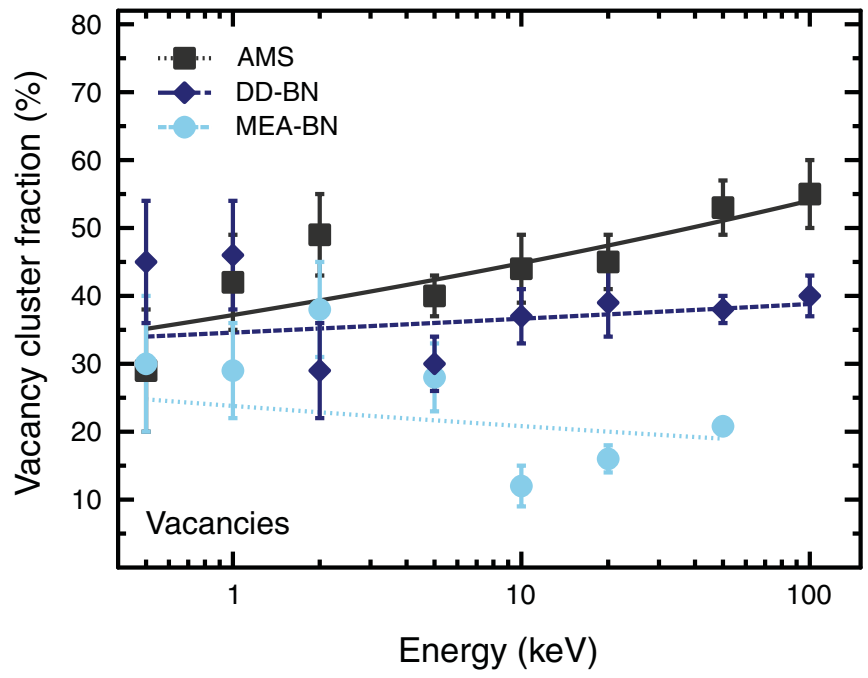

(b)

FIG. 1. (Color online) The (a) interstitial and (b) vacancy cluster fraction in the primary cascade damage. Results obtained from simulations using three different interatomic potentials are shown.

in the high-energy cascades in Ref. 14 either. In this work, we compare the long-term damage obtained from MD simulations with three recent iron potentials, using KMC. These potentials have shown an overall agreement concerning the total amount of defects and the amount of clustered defects, ${ }^{15}$ but differ when it comes to the formation of large clusters. Thus we address the influence of these clusters on damage evolution using different $\mathrm{KMC}$ parameter sets. In the following section, we present our MD simulation results. Next, the KMC model is described, and the last section discusses the implications of the results obtained from these simulations.

\section{MD SIMULATIONS OF COLLISION CASCADES IN FE}

In this study, recoil cascades with PKA energy up to $100 \mathrm{keV}$ were simulated with the MD code PARCAS, ${ }^{16}$ using three different interatomic potentials: One developed by Ackland, Mendelev, and Srolovitz et al. (here denoted AMS), ${ }^{17}$ one from Dudarev and Derlet with short range potential fit by Björkas and Nordlund (DD-BN), ${ }^{15,18}$ and one developed by Müller, Erhart, and Albe with short range part by Björkas and Nordlund (MEA-BN). ${ }^{15,19}$ The largest simulation boxes were of size $150 \times 150 \times 150 a_{0}$, where $a_{0}$ is the lattice parameter for $\mathrm{Fe}$, and the temperature of the border regions was controlled to $300 \mathrm{~K}$. No electronic stopping was applied and periodic boundary conditions were used. At least 10 cascades with randomly chosen recoil directions were simulated for each energy and potential. (The exceptions are the AMS $50 \mathrm{keV}$ case, where 14 cascades were done, and the MEA-BN $100 \mathrm{keV}$ case, where no cascades were done due to high computational cost.) Each simulation was allowed to evolve for 40 ps.

Vacancies and interstitials were identified using WignerSeitz cells. Vacancies within the second-nearest-neighbor distance were considered to belong to the same cluster, and the same was true for interstitials within the third-nearest-neighbor distance. More details about the simulations and analysis are found in Refs. 15 and 20.

The fraction of self-interstitials and vacancies in clusters after the collision cascade as a function of recoil energy for the three potentials studied is presented in Fig. 1. Interstitial fractions increase with increasing recoil energy, and are similar for the different potentials. However, vacancy fractions at high energies differ. This is, as discussed in Refs. 21 and 15, probably related to the speed of the recrystallization front determined by the melting point of the potential. A higher melting point means a faster recrystallization front, which leaves less time for the vacancies to be pushed to the center of the melted zone, and they are therefore left behind in the lattice as single defects. The melting point of MEA-BN is about $2300 \mathrm{~K}$, which is higher than in DD-BN $(\sim 2125 \mathrm{~K})$ and AMS $(\sim 1750 \mathrm{~K})$. The experimental melting point is $1811 \mathrm{~K}^{22}$ More details about defects and basic bulk properties for each potential are found in Table I.

TABLE I. Defect energetics and basic properties of bcc iron according to the three different potentials used in this work. All energies are expressed in eV. $a_{o}$ : lattice constant, $E_{\text {coh }}$ : cohesive energy, $E_{f}$ : formation energy, $E_{\text {mig }}$ : migration energy, $E_{d}^{\text {min }}$ : minimum energy for creating a defect, $V$ : vacancy, and $I$ : interstitial.

\begin{tabular}{|c|c|c|c|c|c|c|c|c|c|}
\hline Potential & $E_{\text {coh }}$ & $a_{0}(\AA)$ & $E_{f}^{I}\langle 110\rangle$ & $E_{f}^{I}\langle 111\rangle$ & $E_{f}^{I}\langle 100\rangle$ & $E_{f}^{V}$ & $E_{\mathrm{mig}}^{I}$ & $T_{\text {melt }}(\mathrm{K})$ & $E_{d}^{\min }$ \\
\hline AMS & 4.013 & 2.86 & 3.67 & 4.12 & 4.45 & 1.71 & $0.28,0.31^{\mathrm{a}}$ & $1750 \pm 25$ & 17 \\
\hline DD-BN & 4.316 & 2.86 & 3.48 & 3.91 & 4.22 & 1.97 & 0.19 & $2125 \pm 25$ & 17 \\
\hline MEA-BN & 4.178 & 2.89 & 4.52 & 4.75 & 5.78 & 1.56 & 0.13 & $2300 \pm 25$ & 16 \\
\hline
\end{tabular}

${ }^{\mathrm{a}}$ Reference 23 . 
The self-interstitial cluster-size distribution for each of the interatomic potentials is plotted in Fig. 2. The distributions are clearly PKA energy dependent for all potentials, and the largest clusters are produced at 50 and $100 \mathrm{keV}$ energies. This result is significantly different from that reported in Ref. 14. Note that the interatomic potential used in those studies was a different one than those reported here.

When comparing the cluster-size distribution for the $50 \mathrm{keV}$ cases separately, the differences in the interatomic potentials are distinct: The fraction of single defects is the smallest in the MEA-BN potential, in favor of small clusters. The AMS has a high fraction of single interstitials but also a long tail of large clusters.

Moreover, AMS produces clusters consisting of over 100 defects, whereas MEA-BN fails to produce clusters over size 30; see Fig. 3. The statistics is, of course, not good enough to state that large clusters will never form in cascades when

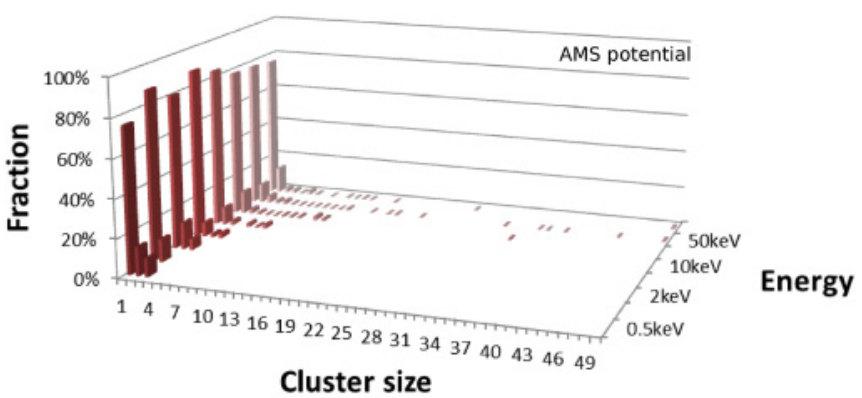

(a) AMS

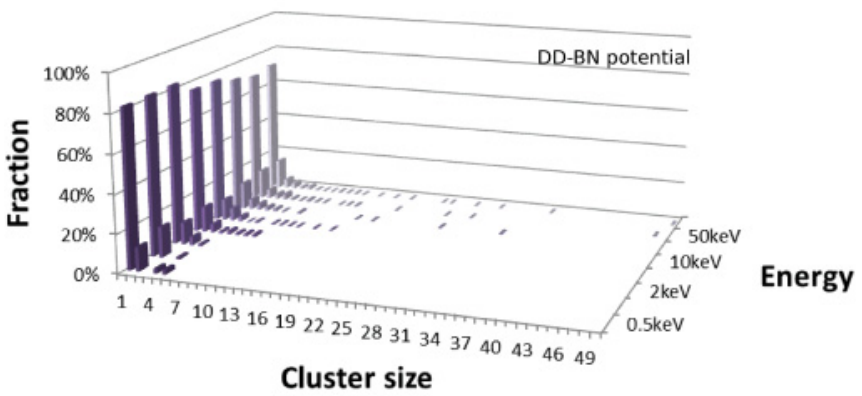

(b) $\mathrm{DD}-\mathrm{BN}$

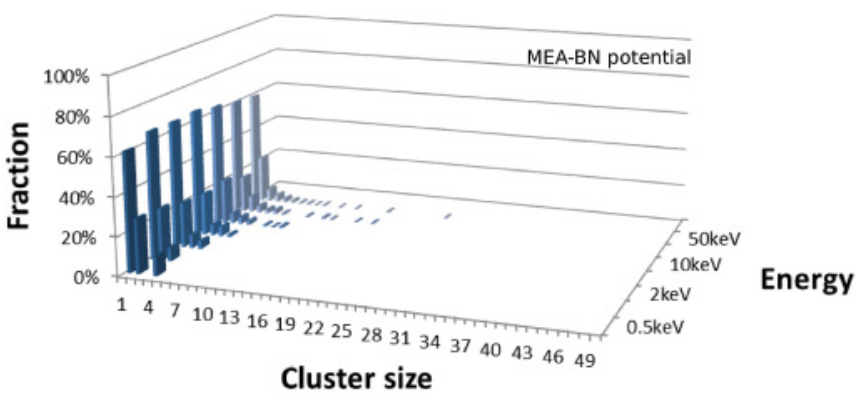

(c) MEA - BN

FIG. 2. (Color online) The size distribution of interstitial defect clusters after collision cascades using different interatomic potentials and different recoil energies. The size 50 data points include clusters of size $\geqslant 50$, and panel (c) does not contain any results for the $100 \mathrm{keV}$ case, as these simulations were not performed with the MEA-BN potential. using MEA-BN, but these simulations indicate that it is less likely. Interestingly, the AMS potential, which has the lowest melting point, is the one with larger self-interstitial clusters, probably due to the formation of larger displacement regions during the collision phase of the cascade.

These differences are not as pronounced in the $100 \mathrm{keV}$ cascades due to the formation of small cascade regions as a consequence of subcascade splitting. Huge clusters in the AMS potential have also been reported in Ref. 24.

The structure and orientation of the largest clusters will be analyzed in more detail and reported elsewhere.

The total number of Frenkel pairs (FPs), with one vacancy and one interstitial, and the fraction of defects in clusters of any size and in clusters larger than 55 are listed in Table II. Note that as already pointed out in previous works, ${ }^{1,15}$ the average number of FPs and self-interstitials in clusters is basically the same for all potentials. There are, however, significant differences in terms of the number of vacancies in clusters, with the MEA-BN showing the least amount of vacancies in clusters and AMS showing the most. Moreover, the AMS potential is the only one that produces large clusters, although with low probability.

\section{KMC SIMULATIONS FOR DAMAGE ACCUMULATION}

The damage distribution, in the form of defect clusters or single defects, obtained from MD using different interatomic potentials was used to study long time evolution with the kinetic Monte Carlo code BIGMAC. ${ }^{25}$ The KMC algorithm applied to radiation damage has been explained in detail in several publications. ${ }^{10,26}$ Briefly, the KMC method is based on the residence time algorithm ${ }^{27}$ and follows the evolution in time of a set of objects given their rates to perform different actions. In our case, the objects are defects (vacancies and self-interstitials of different sizes) and the actions are defect migration or dissociation (association) of a defect from (to) a cluster of defects. The probabilities of migration or dissociation follow an Arrhenius law,

$$
\Gamma=\Gamma_{0} \exp ^{-E / k T},
$$

where $E$ is either the migration energy of a defect or the dissociation energy of a defect from a cluster. The dissociation energy is the sum of the binding energy of a defect to a cluster and the migration energy of that defect. $\Gamma_{0}$ is the jump frequency. For dissociation, this jump frequency is multiplied by the number of defects in a cluster to consider that all defects have the same probability to dissociate from the cluster. Introducing a cascade was considered as an event with a rate corresponding to the desired dose rate, and cascades were chosen randomly from the available MD set and then placed randomly into the simulation box. For further details, see Ref. 10.

In Table III, all values of migration energies and dissociation energies used in the calculations presented in this paper are given. Currently, the migration and stability of small vacancy and self-interstitial clusters in $\alpha-\mathrm{Fe}$ is understood (e.g., Refs. 28 and 29), and values obtained from $a b$ initio calculations have been validated through a comparison with resistivity recovery experiments of electron-irradiated $\mathrm{Fe}^{28}$ However, many questions still remain regarding 


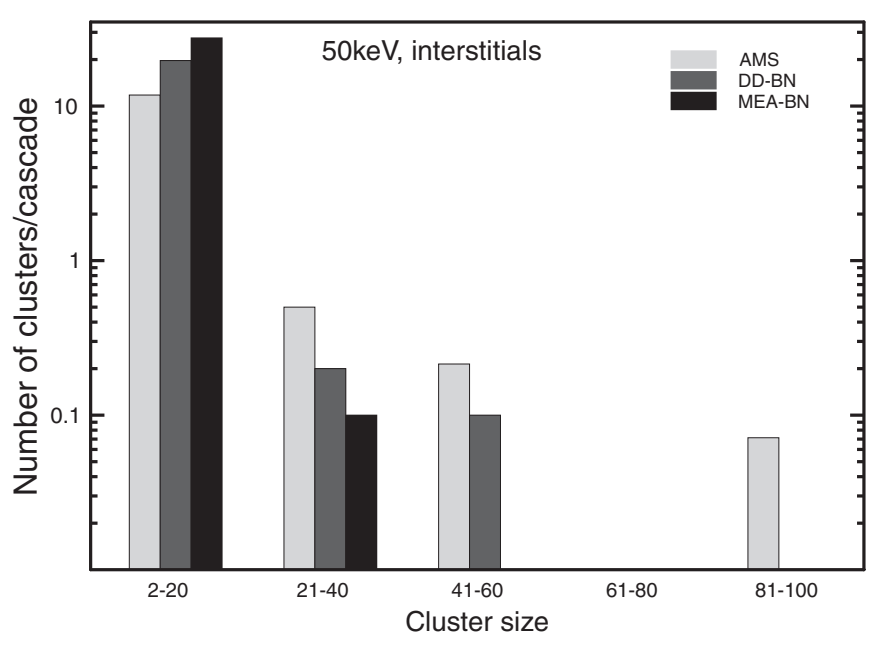

(a)

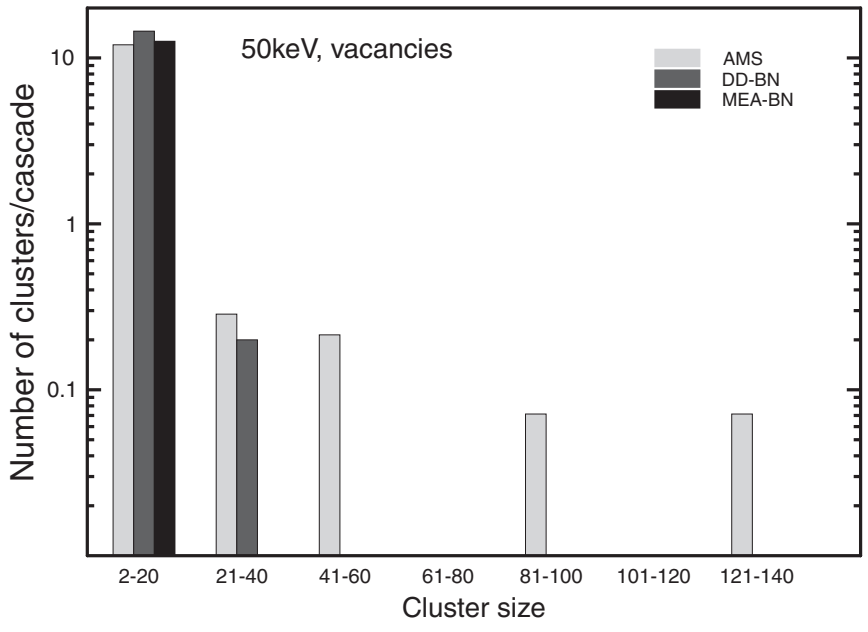

(b)

FIG. 3. The number of (a) interstitial and (b) vacancy clusters of different sizes in the MD $50 \mathrm{keV}$ cascades. Results from simulations with three different potentials are shown.

self-interstitial cluster mobility for sizes that are visible under TEM. While some experiments point to activation energies of self-interstitial clusters of over $1 \mathrm{eV},{ }^{30}$ simulations predict that these clusters should move almost athermally. ${ }^{31}$ Moreover, experiments show the presence of $\langle 100\rangle$ loops in irradiated $\alpha$ Fe under many different conditions of irradiation (electrons or ions), temperatures, and doses, ${ }^{30,32,33}$ even though $\langle 111\rangle$ loops are the lowest energy configuration. At high temperatures $\left(T>300^{\circ} \mathrm{C}\right)$, the transformation of $\langle 111\rangle$ loops into $\langle 100\rangle$ loops has been observed experimentally ${ }^{30}$ and explained theoretically. ${ }^{34,35}$ Other explanations for the presence of $\langle 100\rangle$ loops even at low temperature point to the formation of both $\langle 111\rangle$ and $\langle 100\rangle$ loops in the collision cascade and the growth of these loops by coalescence with small mobile clusters. ${ }^{33}$ Note, however, that $\langle 100\rangle$ loops are also observed under low-temperature electron irradiation. ${ }^{30}$

All of these open questions result in an incomplete model for defect evolution in $\alpha-\mathrm{Fe}$, and assumptions on the migration of self-interstitial clusters must be made in order to reproduce experimental observations. For example, some authors assume that after a certain size of a few tens of self-interstitials, these clusters are immobile, ${ }^{31,36}$ while others include trapping sites in the calculation to reduce the mobility of self-interstitial clusters. ${ }^{37}$ Since the purpose of this study is to evaluate the influence of primary damage on defect evolution and not to reproduce a particular experiment, we use several assumptions for self-interstitial migration for comparison.
We performed KMC simulations of damage accumulation of $50 \mathrm{keV}$ cascades using the database from the three different interatomic potentials. The KMC simulation cell was $143 \times 143 \times 143 \mathrm{~nm}$ and periodic boundaries were used in all directions. Simulations continued until a dose of 0.1 displacements per atom (dpa) was reached at a dose rate of $10^{-6} \mathrm{dpa} / \mathrm{s}$. The dose was calculated using the NRT ${ }^{38}$ formula for displacements with an average threshold of $40 \mathrm{eV}^{39,40}$ The calculations presented here are for a temperature of $300 \mathrm{~K}$.

Figure 4(a) shows the total concentration of self-interstitial clusters (clusters with two defects or more) as a function of dose in dpa using the cascade defects resulting from MD simulations with the three different interatomic potentials. In these calculations, we consider that all self-interstitial clusters with more than four defects and all vacancy clusters with more than five defects are immobile, which are the conditions used to reproduce the resistivity recovery curve of irradiated $\mathrm{Fe}^{28}$ Therefore, the only difference between the calculations is the database used for the initial defect distribution. In this case, the concentration of defects obtained with the different interatomic potentials differs only by factor of about three, with the AMS having the most defects and MEA-BN having the least. The same behavior was seen for vacancy clusters.

As explained above, the behavior of large self-interstitial clusters in terms of mobility and growth is still not completely understood and different assumptions are used in the KMC models. We have therefore studied also the case when all

TABLE II. Total number of Frenkel pairs (FPs) and cluster fraction after $50 \mathrm{keV}$ cascades for the different potentials. Large clusters contain 55 or more defects.

\begin{tabular}{lccccc}
\hline \hline Potential & FPs & $\% V$ in clust. & $\% I$ in clust. & $\% V$ in large clust. & $\% I$ in large clust. \\
\hline AMS & $130 \pm 7$ & $53 \pm 4$ & $64 \pm 3$ & $12 \pm 6$ & $6 \pm 4$ \\
DD-BN & $131 \pm 6$ & $38 \pm 2$ & $63 \pm 4$ & 0 & 0 \\
MEA-BN & $132 \pm 4$ & $20.8 \pm 1.2$ & $68 \pm 3$ & 0 & 0 \\
\hline \hline
\end{tabular}


TABLE III. Defect migration and dissociation energies used in this work. $E_{m}$ : migration energy, $E_{d}$ : dissociation energy, $E_{f}$ : formation energy, $E_{f}^{V}=2.07$ and $E_{f}^{I}=3.77 \mathrm{eV}, E_{b}$ : binding energy of a cluster with two defects, $E_{b}^{V}=0.30$ and $E_{b}^{I}=0.80 \mathrm{eV}$. All values are from Ref. 28, except for the migration energies of $I(n>4)$ that are from Ref. 31.

\begin{tabular}{|c|c|c|c|c|}
\hline Species & $E_{m}(\mathrm{eV})$ & Prefactor $\left(\mathrm{cm}^{2} \mathrm{~s}\right)$ & Migration type & $E_{d}(\mathrm{eV})$ \\
\hline$V$ & 0.67 & $8.2 \times 10^{-3}$ & $3 \mathrm{D}$ & \\
\hline$V 2$ & 0.62 & $8.2 \times 10^{-3}$ & $3 \mathrm{D}$ & 0.97 \\
\hline$V 3$ & 0.35 & $8.2 \times 10^{-3}$ & $3 \mathrm{D}$ & 1.04 \\
\hline$V 4$ & 0.48 & $8.2 \times 10^{-3}$ & $3 \mathrm{D}$ & 1.29 \\
\hline$V n>4$ & immobile & & & $E_{m}^{n=1}+E_{f}^{V}+\frac{\left(E_{b}^{V}-E_{f}^{V}\right)\left[n^{2 / 3}-(n-1)^{2 / 3}\right]}{2^{2 / 3}-1}$ \\
\hline$I$ & 0.34 & $8.2 \times 10^{-3}$ & $3 \mathrm{D}$ & \\
\hline$I 2$ & 0.42 & $8.2 \times 10^{-3}$ & $3 \mathrm{D}$ & 1.14 \\
\hline 13 & 0.43 & $8.2 \times 10^{-3}$ & $3 \mathrm{D}$ & 1.26 \\
\hline I4 (same as I3) & 0.43 & $8.2 \times 10^{-3}$ & $3 \mathrm{D}$ & 1.26 \\
\hline I $n>4$ & $0.06+\frac{0.11}{n^{1.6}}$ & $3.5 \times 10^{-4}+\frac{1.7 \times 10^{-3}}{n^{1.7}}$ & $1 \mathrm{D}$ & $E_{m}^{n=1}+E_{f}^{I}+\frac{\left(E_{b}^{I}-E_{f}^{I}\right)\left[n^{2 / 3}-(n-1)^{2 / 3}\right]}{2^{2 / 3}-1}$ \\
\hline
\end{tabular}

self-interstitial clusters are mobile, with the values given in Table III and obtained from empirical potential calculations for $\langle 111\rangle$ loops. $^{31}$ As done by other authors, ${ }^{37}$ we have included traps for these mobile self-interstitials with a binding energy of $0.9 \mathrm{eV}$ between the self-interstitial and the trap. A concentration of 100 appm of traps is considered in the calculations. Figure 4(b) shows the total concentration of self-interstitial clusters as a function of dose (including both trapped and free self-interstitial clusters) also for the three interatomic potentials and this condition for cluster mobility. Like in the case above, there are no significant differences between the three potentials, although the total concentrations are very different from those in Fig. 4(a), due to the higher recombination between vacancies and self-interstitials.

In the previous case, saturation only occurs when the defect concentration is high enough for cascade overlap to occur. Here, however, self-interstitial clusters can recombine with defects from their own cascade or from the debris of previous ones. Therefore, direct cascade overlap is not needed to reach saturation. These results are in agreement with those reported by Souidi et al., ${ }^{13}$ that is, there are no significant differences in the total defect concentration for different interatomic potentials.

However, the concentration of defects in larger clusters, namely, those that might be visible under transmission electron microscopy (TEM), shows surprising results. The resolution of a TEM for a self-interstitial loop is about $1 \mathrm{~nm},{ }^{9}$ which corresponds roughly to a cluster of 55 defects. Visible voids of similar radius $(1 \mathrm{~nm})$ are vacancy clusters of more than 350 defects. Using these values as the minimum cluster sizes observable, we compute the concentration of visible clusters for the case when most clusters are immobile [Fig. 5(a); corresponding to Fig. 4(a) above] and for the case when all self-interstitial clusters are mobile [Fig 5(b); corresponding to Fig. 4(b) above]. These figures show that there are very large differences between the different interatomic potentials.

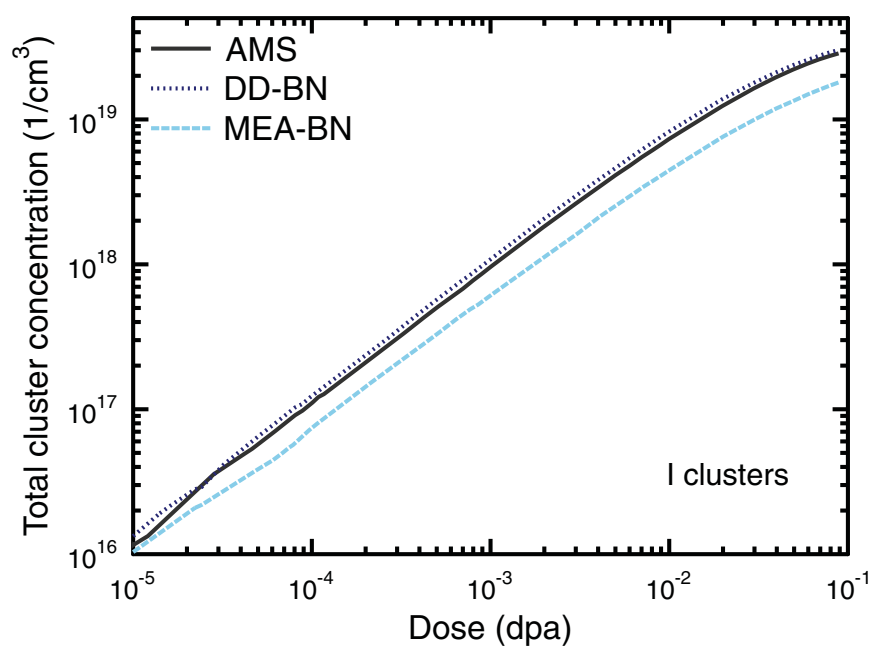

(a)

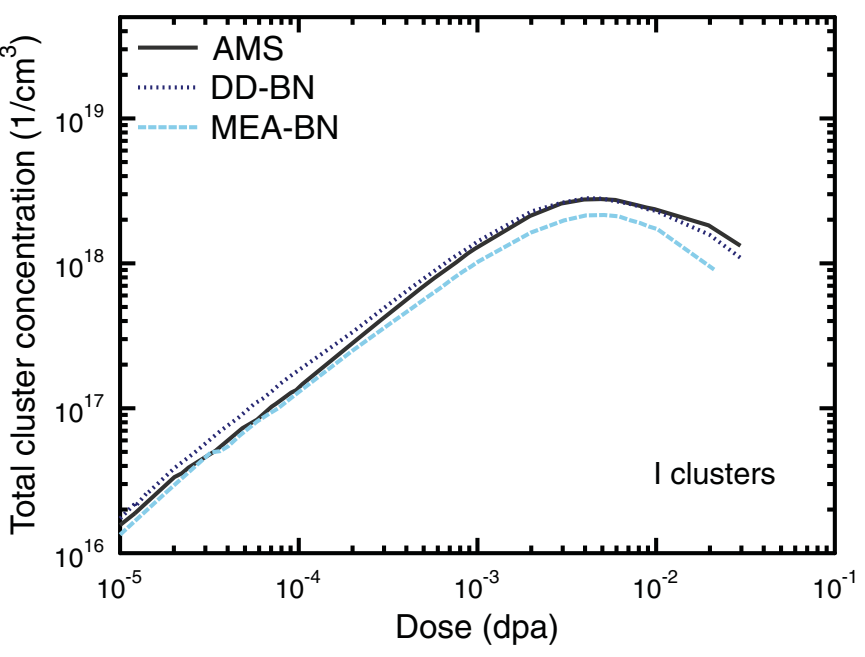

(b)

FIG. 4. (Color online) The total concentration of self-interstitial clusters as a function of dose in the KMC simulations. (a) shows results from the case where only self-interstitials up to size 3 are mobile and (b) shows the case where all self-interstitials mobile and 100 appm traps are included. The primary damage simulated with three different potentials (AMS, DD-BN, MEA-BN) was used as input. 


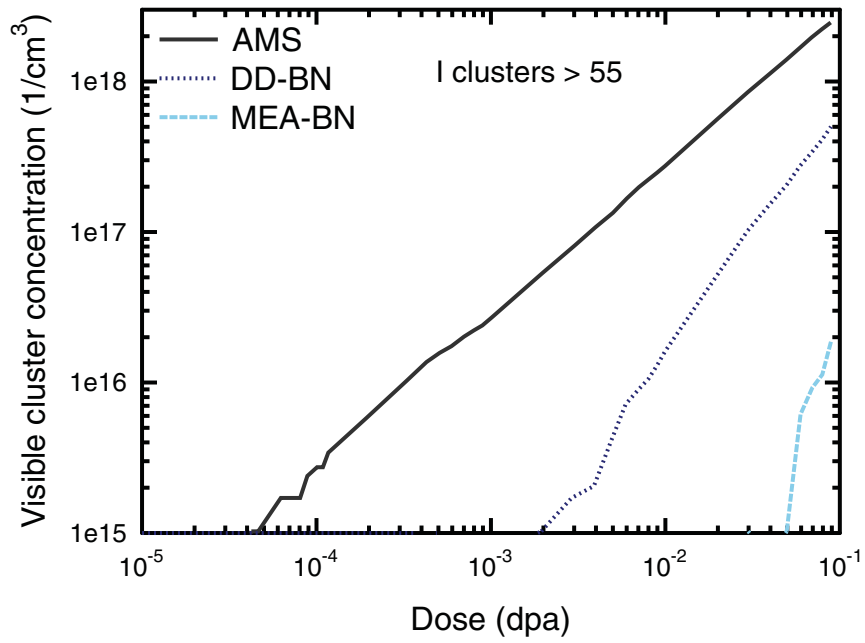

(a)

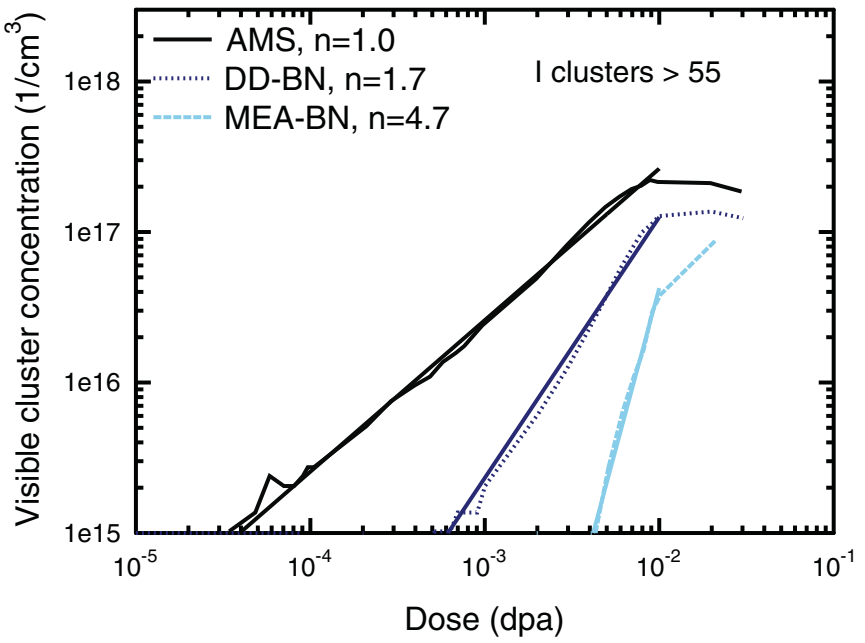

(b)

FIG. 5. (Color online) The concentration of "visible" self-interstitial clusters (more than 55 defects) as a function of dose $\phi$. In case (a), only self-interstitials up to size 3 are mobile, and in (b), all self-interstitials are mobile. In both cases, three different primary damage distributions (modeled with potentials AMS, DD-BN, and MEA-BN) were used as input in the KMC simulations. $n$ is the exponent in the fitting formula $C=\phi^{n}$, where $C$ is the cluster concentration.

Figure 5 shows that the concentration of self-interstitial clusters larger than 55 in the AMS case can be more than three orders of magnitude larger than for the MEA-BN potential at low doses. When traps are included, saturation is reached at around $0.01 \mathrm{dpa}$ and the differences in visible cluster concentration are not large between the different potentials. However, an important difference is the rate of accumulation of visible clusters at low doses. As can be seen in Fig. 5(b) for the AMS potential, the rate of accumulation is slower than for the case of the MEA-BN potential. Fitting these curves to an equation of the type $C=\phi^{n}$, where $C$ is the cluster concentration and $\phi$ is the dose, we obtain a value of $n$ equal to 1.0 for the AMS potential, while for the case of the MEA-BN potential, this value is equal to 4.0. This rate of accumulation can be compared to the values obtained experimentally. For example, irradiation with heavy ions such as Xe gives a slope close to 1 , while irradiation with $\mathrm{Fe}$ ions results in a slope close to 2 for the same energy of the ions. ${ }^{32}$ A slope of 1 indicates that a visible cluster is formed per each additional cascade, as in the case of irradiation with heavy ions. Higher slopes appear when, in order to reach a visible cluster size, coalescence of clusters must occur.

Therefore, these results show that the rate of accumulation obtained with the MEA-BN potential, $n \approx 4$, is too large compared to experimental observations. Also, if the distribution contains very large clusters, the resulting growth rate is too small compared to the experimental values obtained for self-irradiation. One should point out that the $n$ value changes

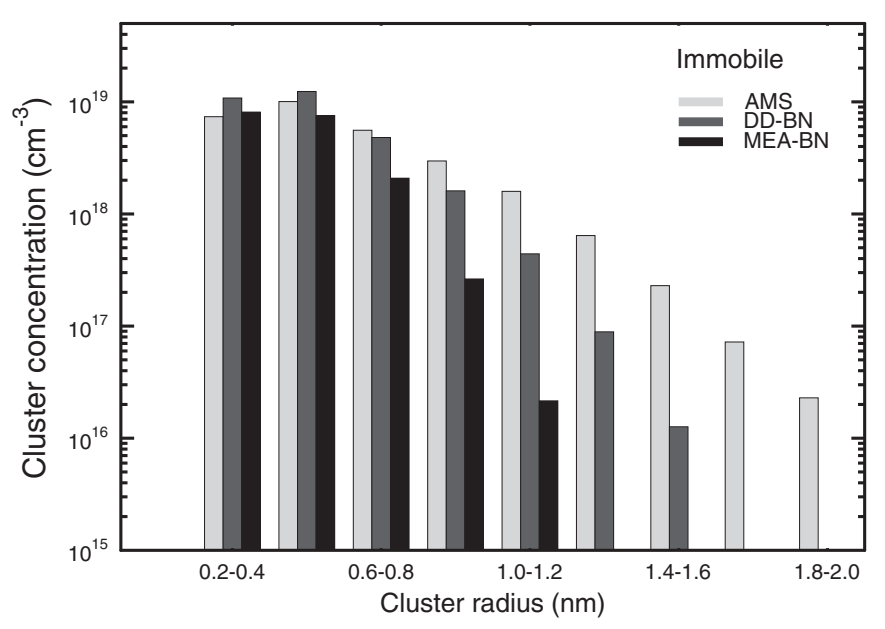

(a)

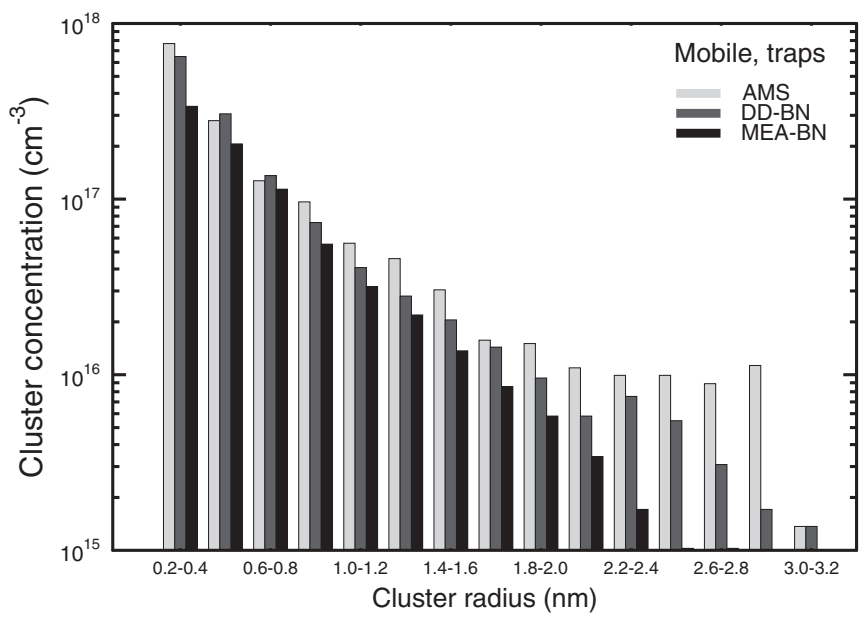

(b)

FIG. 6. The interstitial cluster-size distribution at 0.02 dpa. Data obtained from KMC simulations using different primary damage distributions (modeled with the AMS, DD-BN, and MEA-BN interatomic potentials) are shown. In case (a), only self-interstitials up to size 3 are mobile, and in (b), all self-interstitials are mobile but traps are included. 
when a different cutoff for the smallest cluster size visible is used. For example, if self-interstitial clusters are considered visible when reaching a size of 100 defects, the slope obtained is 2.0, 3.0, and 4.4, for the AMS, DD-BN, and MEA-BN potentials, respectively.

The differences due to the interatomic potential are clearly pronounced when plotting the cluster-size distribution, shown in Figs. 6(a) and 6(b) for the same irradiation dose, which in this case is $0.02 \mathrm{dpa}$. Clearly, the AMS potential has the most and the MEA-BN has the least amount of large clusters.

\section{CONCLUSIONS}

Molecular dynamics simulations using recently developed interatomic potentials for bcc Fe show that there is a dependence of cluster-size distribution on recoil energy, contrary to previous studies. In particular, the maximum cluster size obtained from the simulations increases with recoil energy, at least up to $100 \mathrm{keV}$, when subcascade formation occurs. Moreover, this distribution changes significantly between different interatomic potentials. From the three interatomic potentials studied, the MEA-BN potential is the one that shows the smallest clusters, while the AMS has the largest clusters, both for vacancies and for self-interstitials.

Consequently, the use of cascade sets from different interatomic potentials as input in KMC simulations of damage accumulation results in several orders of magnitude differences in the concentration of clusters visible under TEM. At high doses, when saturation of damage occurs, the differences between the different initial conditions are small. At low doses, however, there is a significant difference in the rate of damage accumulation. When the initial cluster-size distribution presents large clusters, the concentration of visible clusters increases slowly with the dose, while if there are no large clusters present from the initial MD simulations, visible clusters are observed at a much higher dose and the increase in concentration with the dose is much more rapid.

Experimental validation of the defect size distribution obtained in MD after a collision cascade (a few picoseconds) is currently not possible. However, these results show that the comparison of the accumulation rate with the dose of the visible damage can validate the cluster-size distribution obtained from different interatomic potentials. This result can have important consequences when developing predictive models for damage evolution of irradiated $\mathrm{Fe}$ and $\mathrm{Fe}$-based alloys.

The results presented in this paper are for the particular case of damage in $\mathrm{Fe}$, however, the methodology presented to validate cluster-size distributions obtained from MD simulations could be of interest for other metals as well as other fields using ion irradiation, such as the doping of semiconductors.

\section{ACKNOWLEDGMENTS}

The authors wish to thank Max Victoria and Mercedes Hernández-Mayoral for fruitful discussions. This work, supported by the European Communities under the contract of Association between EURATOM/Tekes, was carried out within the framework of the European Fusion Development Agreement (EFDA). It was also supported by the FPVII projects FEMaS and GETMAT, and by the MAT-REMEV Task Agreement of EFDA.
${ }^{1}$ L. Malerba, D. Terentyev, G. Bonny, A. V. Barashev, C. Björkas, N. Juslin, K. Nordlund, C. Domain, P. Olsson, R. Chakarova et al., Proceedings of the ASTM 23rd Symposium on Effects of Radiation on Materials, R. G. Lott and J. T. Busby, eds. (San Jose, CA, June 13-15, 2006) [J. ASTM 11, JAI100692 (2007)].

${ }^{2}$ C. Becquart, A. Souidi, C. Domain, M. Hou, L. Malerba, and R. E. Stoller, J. Nucl. Mater. 351, 39 (2006).

${ }^{3}$ T. Diaz de la Rubia, R. S. Averback, R. Benedek, and W. E. King, Phys. Rev. Lett. 59, 1930 (1987); 60, 76(E) (1988).

${ }^{4}$ C. H. Woo and B. N. Singh, Philos. Mag. A 62, 889 (1992).

${ }^{5}$ B. Singh and S. Zinkle, J. Nucl. Mater. 206, 212 (1993).

${ }^{6}$ A. F. Calder and D. J. Bacon, J. Nucl. Mater. 207, 25 (1993).

${ }^{7}$ W. J. Phythian, R. E. Stoller, A. J. E. Foreman, A. F. Calder, and D. J. Bacon, J. Nucl. Mater. 223, 245 (1995).

${ }^{8}$ Z. Zhou, M. Jenkins, S. Dudarev, A. Sutton, and M. Kirk, Philos. Mag. 86, 4851 (2006).

${ }^{9}$ Z. Zhou, S. Dudarev, M. Jenkins, A. Sutton, and M. Kirk, Proceedings of the Twelfth International Conference on Fusion Reactor Materials (ICFRM-12), (Santa Barbara, CA, Dec 7-12, 2005) [J. Nucl. Mater., Part A 367-370, 305 (2007)].

${ }^{10}$ C. J. Ortiz and M. J. Caturla, J. Comput.-Aid. Mater. Des. 14, 171 (2007).

${ }^{11}$ J. Dalla Torre, C. C. Fu, F. Willaime, A. Barbu, and J.-L. Bocquet, J. Nucl. Mater. 352, 42 (2006).
${ }^{12}$ C. Becquart, C. Domain, L. Malerba, and M. Hou, Nucl. Instrum. Meth. Phys. Res., Sect. B 228, 181 (2005).

${ }^{13}$ A. Souidi, C. Bequart, C. Domain, D. Terentyev, L. Malerba, A. Calder, D. Bacon, R. Stoller, Y. N. Osetsky, and M. Hou, J. Nucl. Mater. 355, 89 (2006).

${ }^{14}$ M. Hou, A. Souidi, C. S. Becquart, C. Domain, and L. Malerba, J. Nucl. Mater. 382, 103 (2008).

${ }^{15}$ C. Björkas and K. Nordlund, Nucl. Instrum. Meth. Phys. Res., Sect. B 259, 853 (2007).

${ }^{16} \mathrm{~K}$. Nordlund, computer code PARCAS (2006). The main principles of the molecular dynamics algorithms are presented in Refs. 41 and 42 . The adaptive time step and electronic stopping algorithms are the same as in Ref. 43.

${ }^{17}$ G. J. Ackland, M. I. Mendelev, D. J. Srolovitz, S. Han, and A. V. Barashev, J. Phys. Condens. Matter 16, S2629 (2004).

${ }^{18}$ S. L. Dudarev and P. M. Derlet, J. Phys. Condens. Matter 17, 1 (2005).

${ }^{19}$ M. Müller, P. Erhart, and K. Albe, J. Phys. Condens. Matter 19, 326220 (2007).

${ }^{20}$ C. Björkas and K. Nordlund, Nucl. Instrum. Meth. Phys. Res., Sect. B 267, 1830 (2009).

${ }^{21}$ K. Nordlund and R. S. Averback, Phys. Rev. B 59, 20 (1999). 
${ }^{22} \mathrm{P}$. Ehrhart, Properties and interactions of atomic defects in metals and alloys, Landolt-Børnstein, New Series, Group III, edited by, H. Ullmaier, Vol. 25 (Springer, Berlin, 1991), Chap. 2, p. 88.

${ }^{23}$ L. Malerba, M. C. Marinica, N. Anento, C. Björkas, H. Nguyen, C. Domain, F. Djurabekova, P. Olsson, K. Nordlund, A. Serra et al. J. Nucl. Mater. 406, 19 (2010).

${ }^{24}$ A. Calder, D. Bacon, A. Barashev, and Y. Osetsky, Philos. Mag. 90, 863 (2010).

${ }^{25}$ M. C. M. D. Johnson and T. Diaz de la Rubia, J. Appl. Phys. 84, 1963 (1998).

${ }^{26}$ C. Becquart, A. Barbu, J. Bocquet, M. Caturla, C. Domain, C.-C. Fu, S. Golubov, M. Hou, L. Malerba, C. Ortiz et al., J. Nucl. Mater. 406, 39 (2010).

${ }^{27}$ M. H. Kalos, Monte Carlo Methods, Vol. I, Basics (Wiley, New York, 1986).

${ }^{28}$ C. Fu, J. Dalla Torre, F. Williame, J.-L. Bocquet, and A. Barbu, Nature Mater. 4, 68 (2005).

${ }^{29}$ C. Marinica and F. Willaime, Solid State Phenom. 129, 67 (2006).

${ }^{30} \mathrm{~K}$. Arakawa, K. Ono, M. Isshiki, K. Mimura, M. Uchikoshi, and H. Mori, Science 318, 956 (2007).

${ }^{31}$ N. Soneda and T. Diaz de la Rubia, Philos. Mag. A 81, 331 (2001).

${ }^{32}$ Z. Yao, M. Hernandez-Mayoral, M. L. Jenkins, and M. A. Kirk, Philos. Mag. 88, 2851 (2008).
${ }^{33}$ Z. Yao, M. Jenkins, and M. Hernandez-Mayoral, Philos. Mag. 90, 4623 (2010).

${ }^{34}$ S. L. Dudarev, R. Bullough, and P. M. Derlet, Phys. Rev. Lett. 100, 135503 (2008).

${ }^{35}$ S. Dudarev, J.-L. Boutard, R. Lässer, M. Caturla, P. Derlet, M. Fivel, C.-C. Fu, M. Lavrentiev, L. Malerba, M. Mrovec et al., J. Nucl. Mater. 386-388, 1 (2009).

${ }^{36}$ M. Caturla, N. Soneda, E. Alonso, B. Wirth, T. Diaz de la Rubia, and J. Perlado, J. Nucl. Mater. 276, 13 (2000).

${ }^{37}$ C. Domain, C. S. Becquart, and L. Malerba, J. Nucl. Mater. 335, 121 (2004).

${ }^{38}$ M. J. Norgett, M. T. Robinson, and I. M. Torrens, Nucl. Eng. Des. 33, 50 (1975).

${ }^{39}$ ASTM Standard E693-94, Standard practice for characterizing neutron exposure in iron and low alloy steels in terms of displacements per atom (dpa) (1994).

${ }^{40}$ K. Nordlund, J. Wallenius, and L. Malerba, Nucl. Instrum. Meth. Phys. Res., Sect. B 246, 322 (2006).

${ }^{41}$ K. Nordlund, M. Ghaly, R. S. Averback, M. Caturla, T. Diaz de la Rubia, and J. Tarus, Phys. Rev. B 57, 7556 (1998).

${ }^{42}$ M. Ghaly, K. Nordlund, and R. S. Averback, Philos. Mag. A 79, 795 (1999).

${ }^{43}$ K. Nordlund, Comput. Mater. Sci. 3, 448 (1995). 